\title{
Long-lasting behavioural consequences of neonatal iron deficiency in high-risk children
}

\section{Abstract}

Little consideration has been given to the long-term consequences of iron deficiency in new-born infants. Fetal iron accretion is compromised by multiple pregnancy complications including preterm birth, gestational diabetes mellitus and fetal growth restriction, while our work has identified increased risks from maternal lifestyle factors such as smoking and obesity for low iron stores at birth. Early-life events, including C-section delivery, also add to this cumulative risk of neonatal iron deficiency, predisposing infants to iron deficiency later in infancy and early childhood. This study aimed to investigate the effect of neonatal iron deficiency on neurological development up to 5 years of age in term-born participants of a maternal-infant birth cohort in Ireland. In the Cork BASELINE Birth Cohort, 697 maternal-infant dyads with prospectively collected lifestyle and clinical data from 15 weeks' gestation had umbilical cord serum ferritin concentrations measured. Neurological assessments were performed at 2 (Bayley Scales of Infant Development and Child Behaviour Checklist [CBCL]) and 5 (Kaufman Brief Intelligence Test and CBCL) years of age. In the cohort, median [IQR] cord ferritin concentrations were $200.9[139.0,265.8] \mu \mathrm{g} / \mathrm{L} ; 7.5 \%$ had neonatal iron deficiency $(<76 \mu \mathrm{g} / \mathrm{L})$. Using the risk factors for neonatal iron deficiency that we previously identified (smoking, obesity, C-section delivery, SGA) in this cohort, as selection criteria, we conducted an a priori sensitivity analysis in 306 children. Of the 306 children identified as high-risk, $12.4 \%$ had neonatal iron deficiency. Those with neonatal iron deficiency had higher median [IQR] CBCL internal (9.0 [5.3,12.0] vs. 5.0 [3.0,10.0]), external $(7.5[4.0,14.8]$ vs. $5.0[2.0,10.0])$ and total problem $(24.5[15.3,40.8]$ vs. $16.0[10.0,30.0]$, all $P<0.05)$ scores at 5 years compared to those without neonatal deficiency. This adverse effect was especially apparent in children of obese mothers $(n=85)$ who were iron deficient at birth, with a total problem score at 5 years of $42.0[24.5,54.5]$ compared to $16.0[8.8,29.3]$ in those not deficient $(P=$ 0.008). Associations were robust to adjustment for confounding factors. No effect on cognition or intelligence at 2 or 5 years was observed in this cohort. This study has identified behavioural consequences of neonatal iron deficiency. Interventions targeting the fetal/neonatal period could, therefore, represent a key opportunity for prevention of iron deficiency and its associated long-term neurological consequences. A dual approach is required, comprising public health strategies targeting prevention, through improving nutrition and health in women, and the development of screening strategies for early detection of iron deficiency in new-borns.

\section{Conflict of Interest}

There is no conflict of interest. 